\title{
Complete genome sequence of Halogeometricum borinquense type strain (PR3 ${ }^{\mathrm{T}}$ )
}

\author{
Stephanie Malfatti ${ }^{1,2}$, Brian J. Tindall ${ }^{3}$, Susanne Schneider ${ }^{3}$, Regine Fähnrich ${ }^{3}$, Alla Lapidus ${ }^{1}$, \\ Kurt LaButtiii , Alex Copeland ${ }^{1}$, Tijana Glavina Del Rio ${ }^{1}$, Matt Nolann ${ }^{1}$, Feng Chen ${ }^{1}$, Susan Lu- \\ cas $^{1}$, Hope Tice ${ }^{1}$, Jan-Fang Cheng ${ }^{1}$, David Bruce ${ }^{1,4}$, Lynne Goodwin ${ }^{1,4}$, Sam Pitluck ${ }^{1}$, lain An- \\ derson ${ }^{1}$, Amrita Pati ${ }^{1}$, Natalia Ivanova ${ }^{1}$, Konstantinos Mavromatis ${ }^{1}$, Amy Chen ${ }^{5}$, Krishna Pa- \\ laniappan ${ }^{5}$, Patrik D'haeseleer ${ }^{1,2}$, Markus Göker ${ }^{3}$, Jim Bristow ${ }^{1}$, Jonathan A. Eisen ${ }^{1,6}$, Victor \\ Markowitz ${ }^{5}$, Philip Hugenholtz ${ }^{1}$, Nikos C. Kyrpides ${ }^{1}$, Hans-Peter Klenk ${ }^{3}$, and Patrick \\ Chain $^{1,2^{*}}$ \\ ${ }^{1}$ DOE Joint Genome Institute, Walnut Creek, California, USA \\ ${ }^{2}$ Lawrence Livermore National Laboratory, Livermore, California, USA \\ ${ }^{3}$ DSMZ - German Collection of Microorganisms and Cell Cultures GmbH, Braunschweig, \\ Germany \\ ${ }^{4}$ Los Alamos National Laboratory, Bioscience Division, Los Alamos, New Mexico, USA \\ ${ }^{5}$ Biological Data Management and Technology Center, Lawrence Berkeley National Labora- \\ tory, Berkeley, California, USA \\ ${ }^{6}$ University of California Davis Genome Center, Davis, California, USA
}

*Corresponding author: Patrick Chain

Keywords: halophile, free-living, non-pathogenic, aerobic, pleomorphic cells, euryarchaeon

Halogeometricum borinquense Montalvo-Rodríguez et al. 1998 is the type species of the ge-
nus, and is of phylogenetic interest because of its distinct location between the halobacterial
genera Haloquadratum and Halosarcina. H. borinquense requires extremely high salt (NaCl)
concentrations for growth. It can not only grow aerobically but also anaerobically using ni-
trate as electron acceptor. The strain described in this report is a free-living, motile, pleomor-
phic, euryarchaeon, which was originally isolated from the solar salterns of Cabo Rojo, Puer-
to Rico. Here we describe the features of this organism, together with the complete genome
sequence, and annotation. This is the first complete genome sequence of the halobacterial
genus Halogeometricum, and this 3,944, 467 bp long six replicon genome with its 3937 pro-
tein-coding and 57 RNA genes is part of the Genomic Encyclopedia of Bacteria and Archaea
project.

\section{Introduction}

Strain PR3 ${ }^{\mathrm{T}}$ (= DSM $11551=$ ATCC $700274=\mathrm{JCM}$ 10706 ) is the type strain of Halogeometricum borinquense, representing the sole species of the genus Halogeometricum [1]. Strain PR3 ${ }^{\mathrm{T}}$ was first described by Montalvo-Rodríguez et al. in 1998 [1] as Gram-stain negative and motile. The organism is of interest because of its position in the tree of life, where it is located between members of the Haloferax/Halorubrum cluster within the large euryarchaeal family Halobacteraceae(Figure 1). Here we present a summary classification and a set of features for $H$. geometricum PR3 ${ }^{\mathrm{T}}$ together with the description of the complete genomic sequencing and annotation.

\section{Classification and features}

In addition to the solar salterns of Cabo Rojo, Puerto Rico, where the type strain PR3 ${ }^{\mathrm{T}}$ and two accompanying strains (PR7 and PR9) were initially isolated [1], the occurrence of strains or phylotypes closely related or belonging to $H$. borinquense have so far only been reported from high salt environments such as an Australian crystallizer pond [6], Maras Salterns in the Peruvian Andes [7], a salt field at Nie, Ishikawa Prefecture, Japan [8], the salterns of Tamilnadu, India (Kannan et al. unpublished), Exportadora del Sal, Guerro Negro, Mexico (FJ609942), a Taiwanese saltern soil (FJ348412), and a low-salt, sulfide- and sulfur-rich spring in southwestern Oklahoma, USA [9]. 
Malfatti, et al.

H. geometricum $\mathrm{PR}^{\mathrm{T}}$ cells are highly pleomorphic (short and long rods, squares, triangles and ovals) and motile by peritrichous flagella (Table 1 and Figure 2). Cells lyse in distilled water. Gas vesicles are present and are responsible for modifying the color of colonies or cell suspensions from red to pink. H. geometricum PR3 ${ }^{\mathrm{T}}$ is aerobic, but also capable of anaerobic growth with nitrate. No anaerobic growth on arginine (arginine dihydrolase is not present). At least $8 \% \mathrm{NaCl}(\mathrm{w} / \mathrm{v})$ is required for growth, reflecting the primary characteristic requirement for high salt concentrations of the Halobacteriaceae [18]. The optimal $\mathrm{NaCl}$ concentration range is $20-25 \% \mathrm{NaCl}(\mathrm{w} / \mathrm{v})$ at $40^{\circ} \mathrm{C}(\mathrm{op}-$ timal growth temperature). Nitrate is reduced to nitrite with the production of gas [1]. Spores or other resting stages have not been reported [1].

H. geometricum PR3 ${ }^{\mathrm{T}}$ is capable of degrading gelatin, but starch is not hydrolysed. A number of sugars and polyols are used as carbon sources, and acid is produced from some sugars [1].

Figure 1 shows the phylogenetic neighborhood of $H$. borinquense strain $\mathrm{PR}^{\mathrm{T}}$ in a $16 \mathrm{~S}$ rRNA based tree. Analysis of the two 16S rRNA gene sequences in the genome of strain PR3 ${ }^{\mathrm{T}}$ indicated that the two genes differ by five nucleotides (nts) from each other, and by 3-5 nts from the previously published 16S rRNA sequence generated from DSM 11551 (AF002984). The slight differences between the genome data and the reported $16 \mathrm{~S}$
rRNA gene sequence are most likely the result of sequencing errors in the previously reported sequence data.

\section{Chemotaxonomy}

The quinone composition of $H$. borinquense strain $\mathrm{PR}^{\mathrm{T}}$ has not been recorded, but based on reports from other members of the family Halobacteriaceae menaquinones with eight isoprenoid units are likely to be present. Typically both MK-8 and MK-8 (VIII- $\mathrm{H}_{2}$ ) are predicted. The lipids are based on isoprenoid diether lipids, but the exact nature of the isoprenoid side chains remains to be investigated. The major phospholipids are the diether, isoprenoid analogs of phosphatidylglycerol and methyl-phosphatidylglycerophosphate (typical of all members of the family Halobacteriaceae), the diether analog of phosphatidyl-glycerol sulfate is absent [1]. A single glycolipid has been reported with an $R_{\mathrm{f}}$ value similar to that of the triglycosyl diether from Haloarcula marismortui, but its structure has not been determined [1]. The pigments responsible for the red color of the cells have not been determined, but it may be predicted that they are carotenoids, probably bacterioruberins. Outer cell layers are probably proteinaceous. The presence of peptidoglycan has not been investigated, but is generally absent from members of this family Halobacteriaceae.

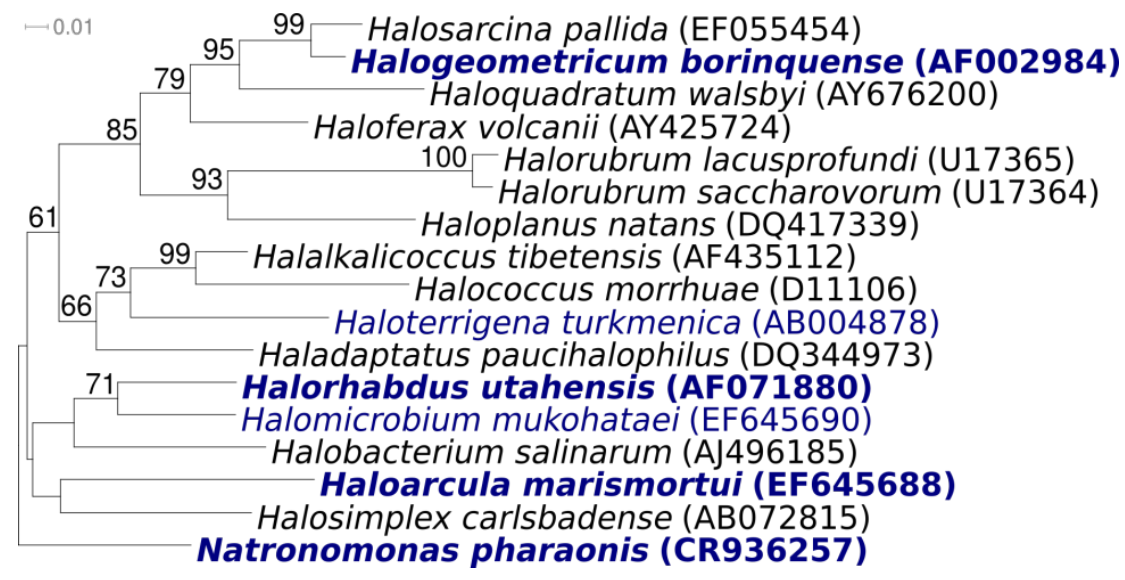

Figure 1. Phylogenetic tree of $H$. borinquense PR $3^{\top}$ with a selection of type strains of the family Halobacteriaceae, inferred from 1,433 aligned 16S rRNA characters [2] under the maximum likelihood criterion [3,4]. The tree was rooted with Natronomonas pharaonsis, the deepest branching member of the family Halobacteriaceae. The branches are scaled in terms of the expected number of substitutions per site. Numbers above branches are support values from 1,000 bootstrap replicates. Strains with a genome sequencing project registered in GOLD [5] are printed in blue; published genomes in bold. 


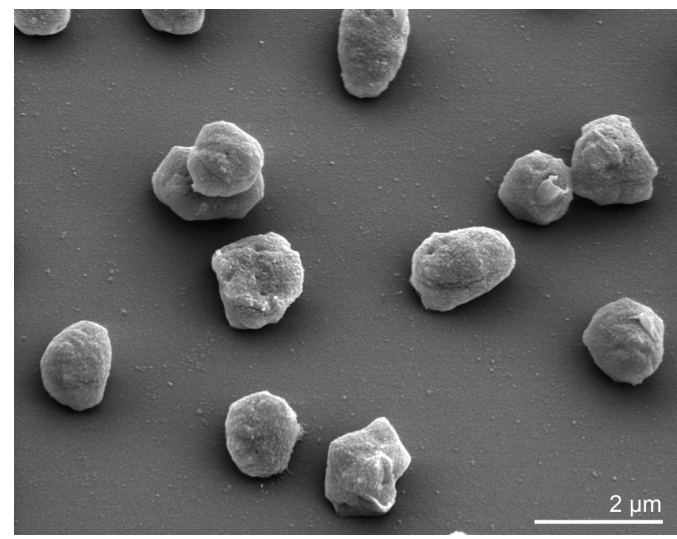

Figure 2. Scanning electron micrograph of $H$. borinquense $\mathrm{PR} 3^{\top}$ (Manfred Rohde, Helmholtz Centre for Infection Research, Braunschweig)

Table 1. Classification and general features of $H$. borinquense $P R 3^{\top}$ according to the MIGS recommendations [10]

\begin{tabular}{|c|c|c|c|}
\hline MIGS ID & Property & Term & $\begin{array}{c}\text { Evidence } \\
\text { code }\end{array}$ \\
\hline & \multirow{8}{*}{ Current classification } & Domain Archaea & TAS [11] \\
\hline & & Phylum Euryarchaeota & TAS [12] \\
\hline & & Class Halobacteria & TAS [13] \\
\hline & & Order Halobacteriales & TAS [14] \\
\hline & & Family Halobacteriaceae & TAS [15] \\
\hline & & Genus Halogeometricum & TAS [1] \\
\hline & & Species Halogeometricum borquinense & TAS [1] \\
\hline & & Type strain PR3 & TAS [1] \\
\hline & Gram stain & negative & TAS [1] \\
\hline & Cell shape & highly pleomorphic & TAS [1] \\
\hline & Motility & motile & TAS [1] \\
\hline & Sporulation & non-sporulating & NAS \\
\hline & Temperature range & mesophile, between $22^{\circ} \mathrm{C}$ and $50^{\circ} \mathrm{C}$ & TAS [1] \\
\hline & Optimum temperature & $40^{\circ} \mathrm{C}$ & TAS [1] \\
\hline & Salinity & halophile, at least $8 \%(w / v) \mathrm{NaCl}$ & TAS [1] \\
\hline \multirow[t]{3}{*}{ MIGS-22 } & Oxygen requirement & $\begin{array}{l}\text { primarily aerobic; facultatively anaerobic } \\
\text { growth via nitrate reduction }\end{array}$ & TAS [1] \\
\hline & Carbon source & $\begin{array}{l}\text { glucose, mannose, fructose, xylose, maltose, } \\
\text { trehalose, cellobiose, raffinose, glycerol }\end{array}$ & TAS [1] \\
\hline & Energy source & carbohydrates & TAS [1] \\
\hline MIGS-6 & Habitat & aquatic & TAS [1] \\
\hline MIGS-15 & Biotic relationship & free living & NAS \\
\hline \multirow[t]{3}{*}{ MIGS-14 } & Pathogenicity & none & NAS \\
\hline & Biosafety level & 1 & TAS [16] \\
\hline & Isolation & solar salterns of Cabo Rojo, Puerto Rico & TAS [1] \\
\hline MIGS-4 & Geographic location & Cabo Rojo, Puerto Rico & TAS [1] \\
\hline MIGS-5 & Sample collection time & 1994 & TAS [1] \\
\hline MIGS-4.1 & Latitude / Longitude & $18,088 /-67,147$ & TAS [1] \\
\hline MIGS-4.2 & & & \\
\hline MIGS-4.3 & Depth & not reported & \\
\hline MIGS-4.4 & Altitude & sea level & NAS \\
\hline
\end{tabular}

Evidence codes - IDA: Inferred from Direct Assay (first time in publication); TAS: Traceable Author Statement (i.e., a direct report exists in the literature); NAS: Non-traceable Author Statement (i.e., not directly observed for the living, isolated sample, but based on a generally accepted property for the species, or anecdotal evidence). These evidence codes are from the Gene Ontology project [17]. If the evidence code is IDA then the property was directly observed for a living isolate by one of the authors or an expert mentioned in the acknowledgements. 
Genome sequencing and annotation Genome project history

This organism was selected for sequencing on the basis of each phylogenetic position, and is part of the Genomic Encyclopedia of Bacteria and Archaea project. The genome project is deposited in the
Genome OnLine Database [5]. The complete genome sequence has not yet been released from GenBank. Sequencing, finishing and annotation were performed by the DOE Joint Genome Institute (JGI). A summary of the project information is shown in Table 2.

Table 2. Genome sequencing project information

\begin{tabular}{|c|c|c|}
\hline MIGS ID & Property & Term \\
\hline MIGS-31 & Finishing quality & Finished \\
\hline MIGS-28 & Libraries used & $\begin{array}{l}\text { Two genomic libraries: } 8 \mathrm{~kb} \text { pMCL200 } \\
\text { and fosmid pcc1Fos Sanger libraries. } \\
\text { One } 454 \text { pyrosequence standard library. }\end{array}$ \\
\hline MIGS-29 & Sequencing platforms & ABI3730, 454 GS FLX \\
\hline MIGS-31.2 & Sequencing coverage & $9.7 \times$ Sanger; $21.8 \times$ pyrosequencing \\
\hline MIGS-30 & Assemblers & Newbler, PGA \\
\hline \multirow[t]{6}{*}{ MIGS-32 } & Gene calling method & $\begin{array}{l}\text { GeneMark 4.6b, tRNAScan-SE-1.23, infer- } \\
\text { nal } 0.81\end{array}$ \\
\hline & INSDC / Genbank ID & СР001688 \\
\hline & Genbank Date of Release & September 10, 2009 \\
\hline & GOLD ID & Gc01108 \\
\hline & NCBI project ID & 20743 \\
\hline & Database: IMG-GEBA & 2501416934 \\
\hline \multirow[t]{2}{*}{ MIGS-13 } & Source material identifier & DSM 11551 \\
\hline & Project relevance & Tree of Life, GEBA \\
\hline
\end{tabular}

\section{Growth conditions and DNA isolation}

H. borinquense PR3 ${ }^{\mathrm{T}}$, DSM 11551, was grown in DSMZ medium 372 (Halobacteria Medium) at $35^{\circ} \mathrm{C}$ [19]. DNA was isolated from 1-1.5 g of cell paste using a Qiagen Genomic 500 DNA Kit (Qiagen, Hilden, Germany) with a modified protocol for cell lysis, LALMP procedure according to $\mathrm{Wu}$ et al. [20]..

\section{Genome sequencing and assembly}

The genome was sequenced using a combination of Sanger and 454 sequencing platforms. All general aspects of library construction and sequencing performed at the JGI can be found at http://www.jgi.doe.gov/. 454 Pyrosequencing reads were assembled using the Newbler assembler version v 2.0.0 (Roche). Large Newbler contigs were broken into 4,435 overlapping fragments of $1,000 \mathrm{bp}$ and entered into assembly as pseudoreads. The sequences were assigned quality scores based on Newbler consensus q-scores with modifications to account for overlap redundancy and adjust inflated q-scores. A hybrid 454/Sanger as- sembly was made using the PGA assembler. Possible mis-assemblies were corrected and gaps between contigs were closed by custom primer walks from sub-clones or PCR products. A total of 2,826 Sanger finishing reads were produced. The error rate of the completed genome sequence is less than 1 in 100,000. Together all sequence types provided $31.5 \times$ coverage of the genome.

\section{Genome annotation}

Genes were identified using GeneMark [21] as part of the genome annotation pipeline in the Integrated Microbial Genomes Expert Review (IMG-ER) system [22], followed by a round of manual curation using the JGI GenePRIMP pipeline [23]. The predicted CDSs were translated and used to search the National Center for Biotechnology Information (NCBI) nonredundant database, UniProt, TIGRFam, Pfam, PRIAM, KEGG, COG, and InterPro databases. The tRNAScanSE tool [24] was used to find tRNA genes, whereas ribosomal RNAs were found by using the tool RNAmmer [25]. Other non coding RNAs were identified by searching the ge- 
nome for the Rfam profiles using INFERNAL (v0.81) [26]. Additional gene prediction analysis and manual functional annotation was performed within the Integrated Microbial Genomes (IMG) platform [27].

\section{Metabolic network analysis}

The metabolic Pathway/Genome Database (PGDB) was computationally generated using Pathway Tools software version 12.5 [28] and MetaCyc version 12.5 [29], based on annotated EC numbers and a customized enzyme name mapping file. It has undergone no subsequent manual curation and may contain errors, similar to a Tier 3 BioCyc PGDB [30].

\section{Genome properties}

The genome is 3,944,467 bp long and comprises one main circular chromosome with a $60 \%$ GC content and five plasmids. Of the 3,994 genes predicted, 3,937 were protein coding genes, and 57 RNAs. Thirty seven pseudogenes were also identified. A total of $62 \%$ of the genes were assigned a putative function while the remaining ones are annotated as hypothetical proteins. The properties and the statistics of the genome are summarized in Table 3. The distribution of genes into COGs functional categories is presented in Figure 3 and Table 4. A cellular overview diagram is presented in Figure 4, followed by a summary of metabolic network statistics shown in Table 5.

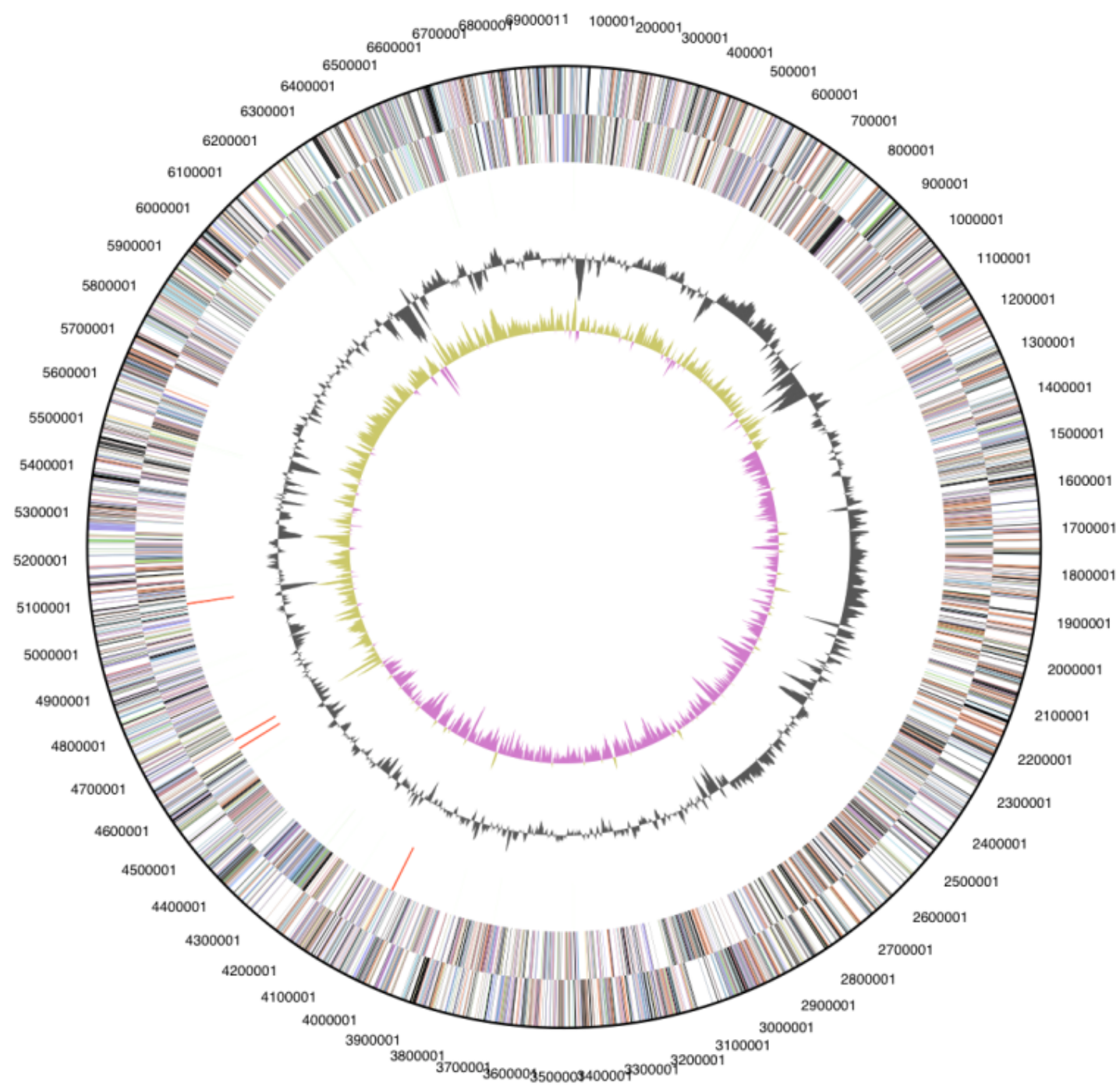

Figure 3. Graphical circular map of the genome. From outside to the center: Genes on forward strand (color by COG categories), Genes on reverse strand (color by COG categories), RNA genes (tRNAs green, rRNAs red, other RNAs black), GC content, GC skew. 
Table 3. Genome Statistics

\begin{tabular}{lrr}
\hline Attribute & \multicolumn{1}{c}{ Value } & \% of Total \\
\hline Genome size (bp) & $3,944,467$ & $100.00 \%$ \\
DNA Coding region (bp) & $3,441,571$ & $87.25 \%$ \\
DNA G+C content (bp) & $2,364,339$ & $59.94 \%$ \\
Number of replicons & 1 & \\
Extrachromosomal elements & 5 & \\
Total genes & 3994 & $100.00 \%$ \\
RNA genes & 57 & $1.90 \%$ \\
rRNA operons & 2 & \\
Protein-coding genes & 3937 & $98.57 \%$ \\
Pseudogenes & 37 & $0.93 \%$ \\
Genes with function prediction & 2486 & $62.24 \%$ \\
Genes in paralog clusters & 741 & $18.55 \%$ \\
Genes assigned to COGs & 2449 & $61.32 \%$ \\
Genes assigned Pfam domains & 2385 & $59.71 \%$ \\
Genes with signal peptides & 533 & $13.35 \%$ \\
Genes with transmembrane helices & 971 & $24.31 \%$ \\
CRISPR repeats & 1 & \\
\hline
\end{tabular}

Table 4. Number of genes associated with the general COG functional categories

\begin{tabular}{lrrl}
\hline Code & Value & \% age & Description \\
\hline J & 162 & 4.1 & Translation, ribosomal structure and biogenesis \\
A & 1 & 0.0 & RNA processing and modification \\
K & 140 & 3.6 & Transcription \\
L & 138 & 3.5 & Replication, recombination and repair \\
B & 3 & 0.0 & Chromatin structure and dynamics \\
D & 0 & 0.1 & Cell cycle control, mitosis and meiosis \\
Y & 0 & 0.0 & Nuclear structure \\
V & 46 & 1.2 & Defense mechanisms \\
T & 113 & 2.8 & Signal transduction mechanisms \\
M & 87 & 2.2 & Cell wall/membrane biogenesis \\
N & 38 & 0.1 & Cell motility \\
Z & 0 & 0.0 & Cytoskeleton \\
W & 0 & 0.0 & Extracellular structures \\
U & 27 & 0.7 & Intracellular trafficking and secretion \\
O & 123 & 3.1 & Posttranslational modification, protein turnover, chaperones \\
C & 174 & 4.4 & Energy production and conversion \\
G & 124 & 3.1 & Carbohydrate transport and metabolism \\
E & 271 & 6.9 & Amino acid transport and metabolism \\
F & 77 & 1.9 & Nucleotide transport and metabolism \\
H & 140 & 3.5 & Coenzyme transport and metabolism \\
I & 98 & 2.5 & Lipid transport and metabolism \\
P & 178 & 4.5 & Inorganic ion transport and metabolism \\
Q & 60 & 1.5 & Secondary metabolites biosynthesis, transport and catabolism \\
R & 433 & 11.0 & General function prediction only \\
S & 227 & 5.8 & Function unknown \\
- & 1488 & 37.8 & Not in COGs \\
\hline
\end{tabular}




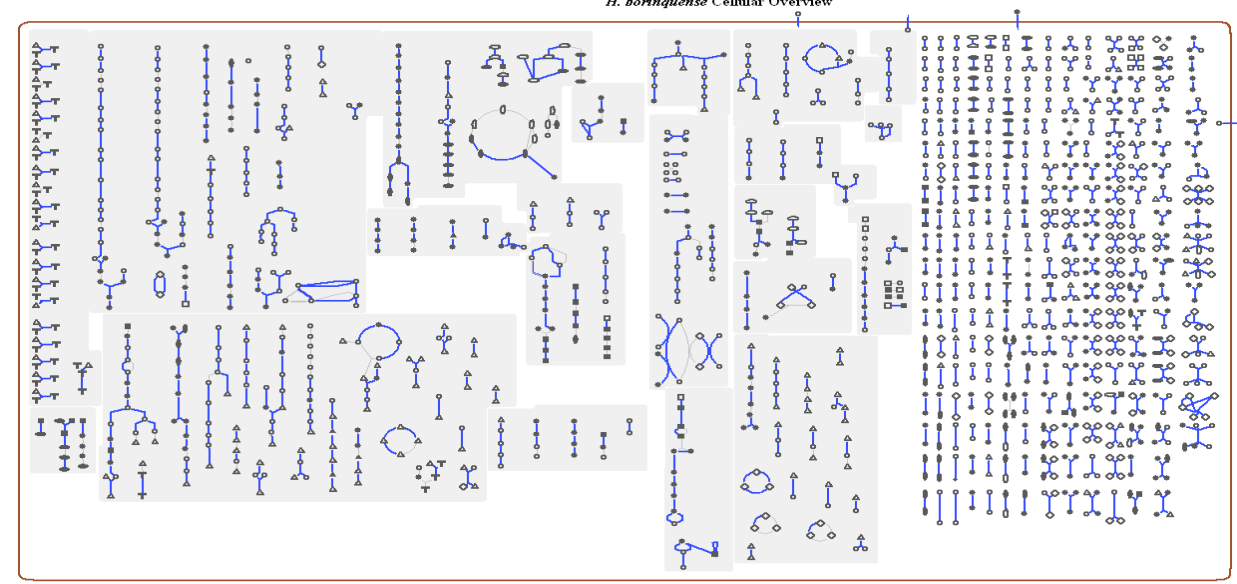

Figure 4. Schematic cellular overview diagram of all pathways of $H$. borinquense strain PR3 ${ }^{\top}$. Nodes represent metabolites, with shape indicating class of metabolite. Lines represent reactions.

Table 5. Metabolic Network Statistics

\begin{tabular}{lr}
\hline Attribute & Valu \\
\hline Total genes & $\mathbf{3 8 0 1}$ \\
Enzymes & 578 \\
Enzymatic reactions & 687 \\
Metabolic pathways & 125 \\
Metabolites & 578 \\
\hline
\end{tabular}

\section{Acknowledgements}

This work was performed under the auspices of the US Department of Energy Office of Science, Biological and Environmental Research Program, and by the University of California, Lawrence Berkeley National Laboratory under contract No. DE-AC02-05CH11231, Lawrence

\section{References}

1. Montalvo-Rodríguez R, Vreeland RH, Oren A, Kessel M, Betancourt C, López-Garriga J. Halogeometricum borinquense gen. nov., sp. nov., a novel halophilic archaeon from Puerto Rico. Int J Syst Bacteriol 1998; 48:1305-1312. PubMed

2. Lee C, Grasso C, Sharlow MF. Multiple sequence alignment using partial order graphs. Bioinformatics 2002; 18:452-464.

PubMed doi:10.1093/bioinformatics/18.3.452

3. Felsenstein J. Evolutionary trees from DNA sequences: a maximum likelihood approach. J $\mathrm{Mol}$ Evol 1981; 17:368-376. PubMed doi:10.1007/BF01734359

4. Stamatakis A, Hoover $\mathrm{P}$, Rougemont J. A rapid bootstrap algorithm for the RAxML web-servers. Syst Biol 2008; 57:758-771. PubMed doi:10.1080/10635150802429642

5. Liolios K, Mavromatis K, Tavernarakis N, Kyrpides NC. The Genomes OnLine Database (GOLD) in 2007: status of genomic and metagenomic projects and their associated metadata. Nucleic Acids Res
Livermore National Laboratory under Contract No. DEAC52-07NA27344, and Los Alamos National Laboratory under contract No. DE-AC02-06NA25396, as well as German Research Foundation (DFG) INST 599/1-1.

2008; 36:D475-D479. PubMed doi:10.1093/nar/gkm884

6. Burns DG, Camakaris HM, Janssen PH, DyallSmith ML. Combined use of cultivation-dependent and cultivation-independent methods indicates that members of most haloarchaeal groups in an Australian crystallizer pond are cultivable. Appl Environ Microbiol 2004; 70:5258-5265. PubMed PubMed doi:10.1128/AEM.70.9.5258-5265.2004

7. Maturrano L, Santos F, Rosselló-Mora R, Antón J. Microbial diversity in Maras Salterns, a hypersaline environment in the Preuvian Andes. Appl Environ Microbiol 2006; 72:3887-3895. PubMed PubMed doi:10.1128/AEM.02214-05

8. Fukushima T, Usami R, Kamekura M. A traditional Japanese-style salt field is a nich for haloarchaeal strains that can survive in $0.5 \%$ salt solution. Saline Systems 2007; 72:3887-3895.

9. Elshahed MS, Najar FZ, Roe BA, Oren A, Dewers TA, Krumholz LR. Survey of archaeal diversity receals an abundance of halophilic archaea in a 
low-salt, sulphide- and sulphur-rich spring. Appl

Environ Microbiol 2004; 70:2230-2239.

PubMed doi:10.1128/AEM.70.4.2230-2239.2004

10. Field D, Garrity G, Gray T, Morrison N, Selengut J, Sterk P, Tatusova T, Thomson N, Allen MJ, Angiuoli SV et al. Towards a richer description of our complete collection of genomes and metagenomes: the "Minimum Information about a Genome Sequence" (MIGS) specification. Nat Biotechnol 2008; 26:541-547. PubMed doi:10.1038/nbt1360

11. Woese CR, Kandler O, Wheelis ML. Towards a natural system of organisms: proposal for the domains Archaea, Bacteria, and Eucarya. Proc Natl Acad Sci USA 1990; 87: 4576-4579.

PubMed doi:10.1073/pnas.87.12.4576

12. Garrity GM, Lilburn TG, Cole JR, Harrison SH, Euzéby J, Tindall BJ. Taxonomic Outline of the Bacteria and Archaea. Release 7.7 March 6, 2007. Michigan State University Board of Trustees. DOI: 10.1601/TOBA7.7.

http://www.taxonomicoutline.org/index.php/toba

13. Grant WD, Kamekura M, McGenity TJ, Ventosa A. Class III. Halobacteria class. nov. In: DR Boone, RW Castenholz, GM Garrity (eds): Bergey's Manual of Systematic Bacteriology, 2nd edition, Vol. 1, The Archaea and the deeply branching and phototrophic Bacteria, Springer-Verlag, New York, 2001.p 294.

14. Grant WD, Larsen H. Group III. Extremely halophilic archaeobacteria. Order Halobacteriales ord. nov. In: JT Staley, MP Bryant, N. Pfennig, JG Holt (eds), Bergey's Manual of Systematic Bacteriology, 1st edition, Vol. 3, The Williams \& Wilkins Co., Baltimore, 1989, pp. 2216-2218.

15. Gibbons NE. Family V. Halobacteriaceae fam. nov. In: RE Buchanan, NE Gibbons (eds): Bergey's Manual of Determinative Bacteriology, 8th edition, The Williams \& Wilkins Co, Baltimore, 1974, p. 269.

16. Anonymous. Biological Agents: Technical rules for biological agents www.baua.de TRBA 466.

17. Ashburner M, Ball CA, Blake JA, Botstein D, Butler $\mathrm{H}$, Cherry JM, Davis AP, Dolinski K, Dwight SS, Eppig JT, et al. The Gene Ontology Consortium. Gene ontology: tool for the unification of biology. Nat Genet 2000; 25:25-29.

PubMed doi:10.1038/75556

18. Norton C. Rediscovering the ecology of halobacteria. ASM News 1992; 58:363-367.
19. List of media used at DSMZ for cell growth: http://www.dsmz.de/microorganisms/media_list.ph $\mathrm{p}$

20. Wu M, Hugenholtz P, Mavromatis K, Pukall R, Dalin E, Ivanova N, Kunin V, Goodwin L, Wu M, Tindall BJ, et al.. A phylogeny-driven genomic encyclopedia of Bacteria and Archaea. Nature 2009 (In press)

21. Besemer J, Lomsadze A, Borodovsky M. GeneMarkS: a self-training method for prediction of gene starts in microbial genomes. Implications for finding sequence motifs in regulatory regions. Nucleic Acids Res 2001; 29:2607-2618. PubMed PubMed doi:10.1093/nar/29.12.2607

22. Markowitz VM, Mavromatis K, Ivanova NN, Chen IMA, Chu K, Kyrpides NC. Expert Review of Functional Annotations for Microbial Genomes. Bioinformatics 2009 25:2271-2278.

23 Pati A, Ivanova N, Mikhailova, N, Ovchinikova G, Hooper SD, Lykidis A, Kyrpides NC. GenePRIMP: A Gene Prediction Improvement Pipeline for microbial genomes. (Submitted) 2009.

24. Lowe TM, Eddy SR. tRNAscan-SE: a program for improved detection of transfer RNA genes in genomic sequence. Nucleic Acids Res 1997; 25:955964. PubMed doi:10.1093/nar/25.5.955

25. Lagesen K, Hallin P, Rødland EA, Staerfeldt HH, Rognes T, Ussery DW. RNAmmer: consistent and rapid annotation of ribosomal RNA genes. Nucleic Acids Res 2007; 35:3100-3108. PubMed doi:10.1093/nar/gkm160

26. Griffiths-Jones S, Moxon S, Marshall M, Khanna A, Eddy SR, Bateman A. Rfam: annotating non-coding RNAs in complete genomes. Nucleic Acids Res 2005; 33:D121-D124. PubMed doi:10.1093/nar/gki081

27. Markowitz VM, Szeto E, Palaniappan K, Grechkin Y, Chu K, Chen IMA, Dubchak I, Anderson I, Lykidis A, Mavromatis K, et al. The Integrated Microbial Genomes (IMG) system in 2007: data content and analysis tool extensions. Nucleic Acids Res 2008; 36:D528-D533. $\underline{\text { PubMed }} \underline{\text { doi:10.1093/nar/gkm846 }}$

28. Karp PD, Paley SM, Romero P. The Pathway Tools Software. Bioinformatics 2002; 18:S225-S232. PubMed

29. Caspi R, Karp P, Foerster H, Fulcher CA, Kaipa P, Krummenacker M, Latendresse M, Paley SM, Rhee SY, Shearer A, et al. The MetaCyc Database of metabolic pathways and enzymes and the BioCyc collection of pathway/Genome Databases. Nucleic 
Acids Res 2008; 36:D623-D631. PubMed

PubMed doi:10.1093/nar/gkm900

30. Karp PD, Ouzounis CA, Moore-Kochlacs C, Goldovsky L, Kaipa P, Ahren D, Tsoka S, Darzentas N,
Kunin V, Lopez-Bigas N. Expansion of the BioCyc collection of pathway/genome databases to 160 genomes. Nucleic Acids Res 2005; 33:6083-6089. PubMed doi:10.1093/nar/gki892 